\title{
Use of SSR Fragment Length Homozygotes for Orangutan Systematics
}

\author{
SREETHARAN KanthasWamy, O. R. P. BININDA-EMONDS, \\ C. WARDEN, J. L. ViraY, and D. G. SMith \\ University of California
}

\begin{abstract}
The use of simple sequence repeat (SSR) loci lacking length polymorphisms among their alleles (defined here as homozygotes) in systematic studies has thus far been largely ignored due to their apparent lack of genetic heterogeneity. However, in this paper we show that point mutations, insertions, and deletions within SSR loci of identical size can be highly informative for population genetic and evolutionary studies. Our preliminary study of the von Willebrand factor (vWF) locus shows that there is a significant level of divergence among the Bornean Orangutans which implies that present orangutan subpopulations in Borneo are genetically isolated from each other. However, our findings do not confirm that the Bornean and Sumatran Orangutans form two separate clades.
\end{abstract}

Key Words: Pongo pygmaeus; Microsatellite polymorphism; vWF; Phylogenetic analysis; Conservation.

\section{INTRODUCTION}

Genetic differentiation in orangutans, mainly that between the Bornean (Pongo pygmaeus pygmaeus) and Sumatran (Pongo pygmaeus abelii) populations, has been previously characterized using allozymes, coding and non-coding mitochondrial $(\mathrm{mt})$ and nuclear DNA, mt rRNA and chromosomal polymorphisms (JANCZEWSKI, 1989; JANCZEWSKI et al., 1990; RYDER \& Chemnick, 1993; Xu \& ARnason, 1996; ZHl et al., 1996). Most of these studies have supported the recognition of both populations as separate species. KANTHASWAMY et al. (in press) pointed out that the relative ease of unequivocally resolving SSR alleles makes these hypervariable loci not only suitable for genetic characterization of orangutans at the species level but also at individual, lineage and population levels. However, there is much contention about whether sufficient substructuring exists among the Bornean Orangutans to warrant reclassifying some populations as separate subspecies. ZHI et al. (1996) addressed this issue using detailed molecular data based upon mtDNA-RFLP, minisatellites, mitochondrial 16S rRNA genes and allozyme analysis, and concluded that there is insufficient genetic differentiation among the Bornean Orangutans to regard any subset of them as a separate subspecies. Given that the estimated genetic distances among populations of orangutans vary with different gene families and molecular systematic techniques, and that potential inferences from such studies may have important management implications for orangutan conservation, more studies using a variety of molecular techniques and loci are necessary. Furthermore, sampling of members of such a highly endangered species is often inadequate due to their low numbers in the wild and regulations imposed by wildlife managers who restrict invasive sampling of the animals. Insufficient sample size can impose serious limitations on the estimation of genetic subdivision especially when less informative molecular markers are employed. Small populations also experience a greater level of inbreeding and population substructuring which exacerbates this problem. 
The conservation of non-coding simple sequence repeat (SSR) loci across closely related taxa (DeKa et al., 1994; BRUford et al., 1996; MEYer et al., 1995) allows for convenient crossspecies amplification of these loci using specific oligonucleotide primers for the polymerase chain reaction (PCR, SAIKI et al., 1988). The successful use of human primers for this purpose has been described for several non-human primate species (MORIN \& WOODRUFF, 1992; BowCOCK et al., 1994; MoRIN et al., 1997; SMITH et al., 1999; ELY et al., in press). SSR loci consist of tandemly repeated motifs (up to 60 times: GoldSTEIN \& Pollock, 1997), each from two (dimeric) to six (hexameric) nucleotides long which are widely dispersed throughout the eukaryotic nuclear genome (TAUTZ, 1989; WEBER \& MAY, 1989). SSR loci are the most mutable of genes with mutation rates of $0.0012 /$ locus/generation in cell lines derived from CEPH families (WeBer \& WONG, 1993) and 0.01 to 0.0001/locus/generation in mice (DALlas, 1992). Polymorphisms in SSRs have been envisioned as resulting from polymerase slippages (saltatory replication), unequal crossovers or possibly gene conversion (LEWIN, 1997), and often exhibit high levels of heritable variation in the number of repeats of a particular motif (AMOS et al., 1993). The variability in the number of repeated motifs in SSR loci provides at least one basis for length variation among its alleles that generates large numbers of heterozygotes in a given population. SSR loci that are length-monomorphic have been hitherto excluded from both evolutionary and population genetic studies due to their apparent lack of genetic variability. This paper describes our preliminary study of the nucleotide sequence in a monomorphic SSR locus, the von Willebrand factor, or vWF, gene (MANCuso et al., 1989), in various Bornean and Sumatran Orangutan populations.

In this study, homozygous individuals are those with two alleles of identical size at the vWF locus. Although the alleles may also have identical origins [i.e. a reflection of generations of inbreeding, population substructuring, bottlenecks or combinations thereof (NICHOLS \& BALDING, 1991)], they need not. The DNA sequences of SSR alleles of identical size may contain considerable variation due to point substitutions providing large amounts of evolutionary data. Such sequences are particularly useful for phylogenetic studies at or below the species level because they can be regarded as selectively neutral, and hence rapidly evolving, and provide a virtually unlimited supply of sequence data. The sequence data of these loci were used to estimate pairwise molecular genetic distance among individual representatives of various populations of Bornean and Sumatran Orangutans to assess taxonomic distinctions among them.

\section{METHODS}

The vWF locus was among nine successfully amplified in orangutans using human primers to characterize Bornean and Sumatran populations as separate species (KANTHASWAMY et al., in press). Although this locus was shown to be monomorphic in length across orangutans and, thus, contributed minimally to conventional SSR genotyping in their study, the authors suspected that $\mathrm{vWF}$ alleles derived from different localities contain sufficient genetic heterogeneity among individuals to elucidate genetic subdivision among the island populations.

The present study sampled 31 (unrelated) Bornean and 25 Sumatran Orangutans. Purified DNA, blood, sera, tissue, and hair samples were shipped from various facilities (see Acknowledgements) on dry ice to the Molecular Anthropology Laboratory, Department of Anthropology, University of California, Davis, CA, USA. DNA was extracted using the Qiagen blood and tissue kits (QIAGEN, Inc., Chatsworth, CA, USA) following the manufacturer's suggested protocol. PCR-based amplification of the $\mathrm{VWF}$ locus for each of the 56 orangutans was conducted using human PCR primers (MapPairs ${ }^{\mathrm{TM}}$, Research Genetics, Huntsville, AL, USA). 
In general, PCRs, electrophoreses and gel imaging of loci were conducted as previously described (MORIN \& SMITH, 1995) with slight modifications (MORIN et al., 1997; KANTHASWAMY \& SMiTH, 1998). To further ensure accurate comparisons of allele sizes between gels, a panel of amplified positive standards was run on every gel. One animal each from East Sabah, Sarawak, Kutai, Gunung Palung, West Central Kalimantan, South Central Kalimantan, Northeast Sabah, West Sabah, and two Sumatran sequences (Sumatra 24 and 26), all homozygous for the same allele at the vWF locus, were selected for sequencing (see Fig. 1).

PCR fragments from each of these individuals were first screened with a heteroduplex mobility analysis (HMA) to confirm that they consisted only of homoduplexes, i.e. the DNA of both parental alleles of each individual comprised perfectly complementary nucleotide acid chains and therefore were identical (DEINARD, A. S., pers. comm.). The remaining portions of PCR product of these 10 samples were purified on Microcon ${ }^{\mathrm{TM}} 100$ microconcentrating columns (Amicon, Inc., Beverly, MA, USA). Both forward and reverse nucleotide sequences were obtained for each amplicon by the DNA Sequencing Facility at the Division of Biological Sciences, University of California, Davis. A BLAST search was used to obtain other primate sequences in the GenBank database that corresponded to the vWF sequence of the orangutan from Gunung Palung. The accession numbers are chimpanzees: X80144, X80145, X80146, X80148, X80149, and X80157; gorilla: X80150; orangutans: X80151 and X80152; rhesus macaques: X80153 and X80154. Sequences derived from this study and those from GenBank were aligned using the Multiple Sequence Alignment on the BCM Search Launcher (http://dot.imgen.bcm.tmc.edu: 9331) and improved by eye.

A maximum parsimony (MP) analysis was conducted using PAUP* 4.0b2 (SwOFFORD, 1999). Because it was perceived that insertions and deletions were potentially phylogenetically informative, they were coded as an additional character state as is usually done in cladistic analysis (KITCHING et al., 1998). Chimpanzee and gorilla sequences were used as outgroups. Searches were conducted using a branch and bound algorithm, which provided an optimal solution.

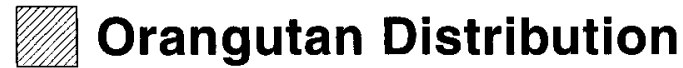
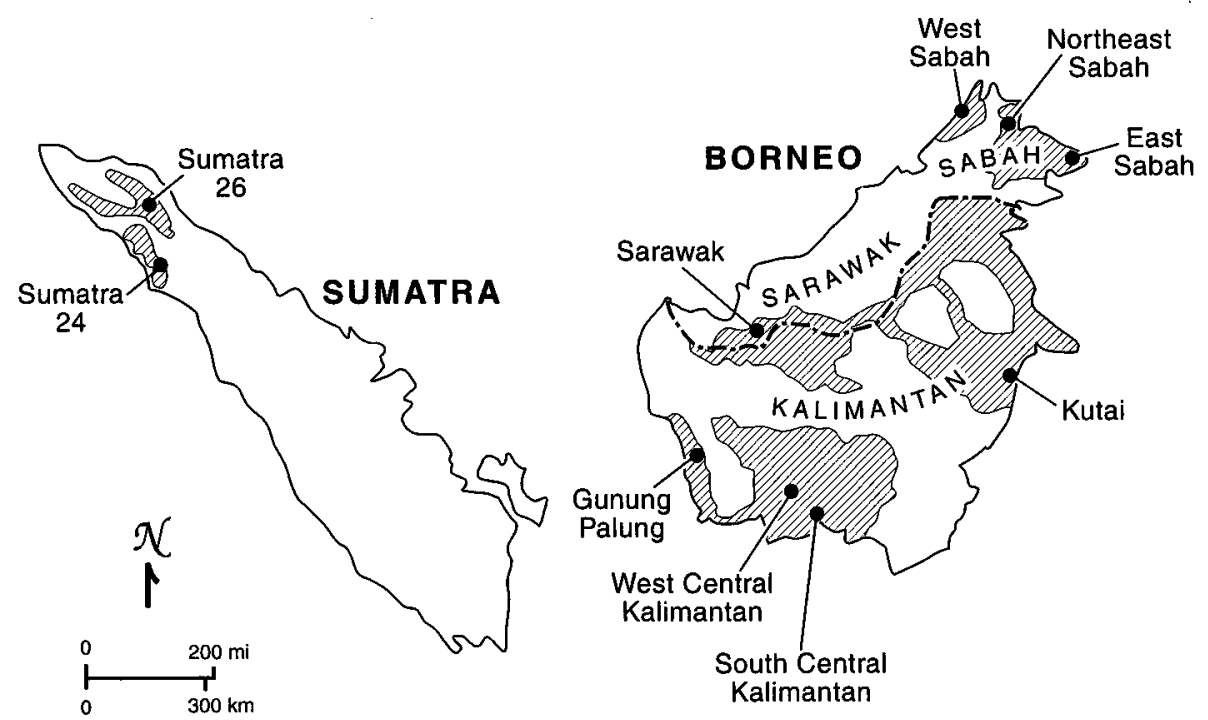

Fig. 1. Orangutan sampling sites in Borneo and Sumatra. 
Confidence intervals were defined by the FELSENSTEIN (1985) bootstrap method. One thousand replicates were used, each employing a heuristic search strategy using the "closest" addition sequence holding ten trees at each step, TBR branch swapping, and unlimited MAXTREES.

\section{RESULTS}

The vWF locus exhibited size polymorphisms in all taxa compared (MEYER et al., 1995; MORIN et al., 1997) in this study but very little fragment size heterozygosity was observed for the orangutans $(n=56)$. Gel electrophoresis of PCR products for this locus revealed single bands of identical length in all orangutans except for individuals from Sarawak, Kutai, and Sumatra 24, each of which appeared to exhibit a single band shorter by one (tetranucleotide) motif than that of the rest. From the HMA, it was inferred that each orangutan in this study exhibited solely homoduplex DNA fragments. Therefore, these individuals are autochthonous and, thus, are representatives of divergent $\mathrm{vWF}$ lineages that may have been genetically isolated from each other for a significant length of time.

The orangutan sequences in our study (Fig. 2) exhibited similar patterns to those described in MEYER et al. (1995), who unfortunately, did not specify the collection localities of their Pongo sequences. That is the hominoid orthologs of the $\mathrm{VWF}$ gene showed variation in the organization of TCTA and TCTG repeats at the 5'end of the repeat array. As shown in the figure, the TCTG and TCTA units alternate in their arrangement in chimpanzees and gorilla. Across the primate genera, there were two tandem TCCA repeat motifs (the conserved TCCATCCA region), some with point mutations, immediately adjacent to the 3'flanking region. The chimpanzee and gorilla sequences showed high frequencies of TCTA repeats (MEYER et al., 1995). As in the GenBank derived orangutan sequences (MEYER et al., 1995), our Pongo sequences lacked several TCTG and TCTA units compared to chimpanzee and gorilla sequences (MOLLER et al., 1994). Overall, across hominoids, the vWF orthologue repeat array sizes appear to have evolved by a stepwise mutational process (GOLDSTEIN \& POLLOCK, 1997) as observed by MEYER et al. (1995). This phenomenon, however, was not very evident in Pongo samples. This species' sequences contained significant polymorphisms, i.e. single nucleotide heterogeneity and degraded or altered repeat units, that may have resulted from random point mutations as well as random insertions and deletions (indels: COLSON \& GOLDSTEIN, 1999); our orangutan phylogenetic analysis was based on these polymorphisms. The rhesus monkey sequences exhibited dramatic differences that led to TCCG and TCCA repeats, and a prevalence of indels in the 5 'flanking region (MEYER et al., 1995).

Phylogenetic analysis revealed clear separation of the African apes from orangutans and appreciable substructuring among orangutans, albeit with fairly weak support as revealed by the bootstrap analysis (Fig. 3). The pattern of relationships is illustrated in a cladogram rather than a dendrogram because there is no gene flow among the taxa (TEMPLETON, 1998). Well supported groups include all orangutans except the GenBank individual X80151 and all African apes. Interestingly, the single gorilla sequence was not well differentiated from the chimpanzee sequences while the orangutan sequences often did not correlate with geography. A notable example is the separation of the two Sumatran sequences into separate (albeit poorly supported) clades, each of which clusters more closely with sequences of Bornean individuals.

Table 1 presents the pairwise mean genetic distances (per nucleotide) obtained among orangutans and other primates; the considerable overlap among the hominoid ranges is noteworthy. The average pairwise distance between the rhesus monkeys and the other hominoid species is $11.9 \%(\mathrm{SD}=2.4 \%$ ) with a range from 8.7 to $19.1 \%$. The mean pairwise distance of the vWF 


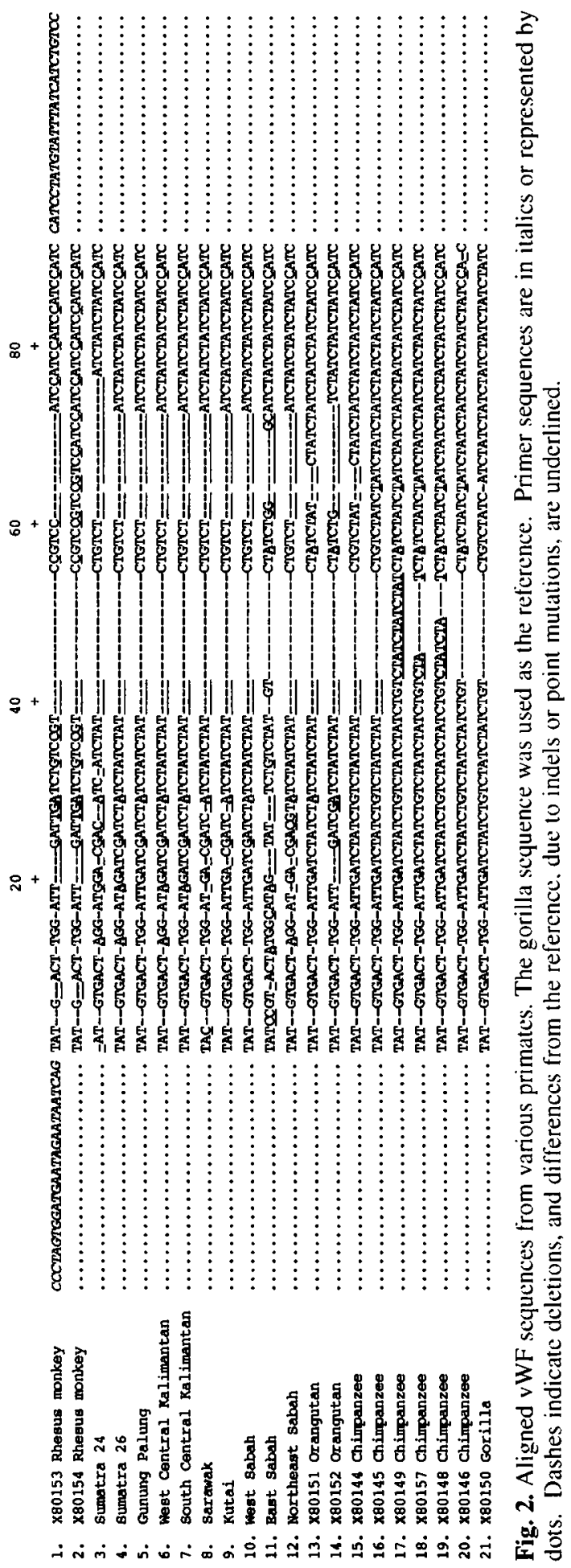


sequence between all pairs of orangutans is $\bar{x}=2.5 \% \pm 1.4 \%$, ranging from 0 to $6.2 \%$ while among the chimpanzees this distance is $\bar{x}=0.4 \% \pm 0.4 \%$ with a range of 0 to $0.4 \%$. When the sequence from East Sabah, which appears deviant from all other orangutan sequences and, perhaps, erroneous, was eliminated, the average pairwise distance among orangutans decreased $(\bar{x}$ $=2.1 \% \pm 1.2 \%$; range $=0$ to $5.0 \%$ ). The orangutan-chimpanzee mean distances ranged from 0.8 to $6.5 \%$ and the gorilla-orangutan comparisons ranged from 6.8 to $11.9 \%$.

\section{DISCUSSION}

The sequence data reveal a previously underutilized class of variation at a monomorphic SSR locus. DNA sequencing might increase the information content of a panel of SSR markers regardless of the fact that these markers are not length-polymorphic in a given species. The DNA sequencing of the primate vWF orthologues provides several insights regarding the evolution of SSR loci in the orangutan and, more generally, in non-human primates.

While the repeat array size in the primate vWF locus vary in a seemingly non-random manner (MeYer et al., 1995), we suggest that the point mutations and indels in the Pongo vWF locus have occurred randomly due to complex non-stepwise mutations, as observed by COLSON and GolDSTEIN (1999) in several polymorphic loci in Drosophila spp. These polymorphisms are the basis of our interpretation of the historical relationships among the different orangutan populations. MEYER et al. (1995) also revealed a correlation between the repeat array size and the phylogenetic distances across the primate taxa at the locus. Here, despite occurring to a lesser degree, this correlation is again observed among the representative orangutan sequences. Furthermore, the between individual differences in repeat array size among orangutans are

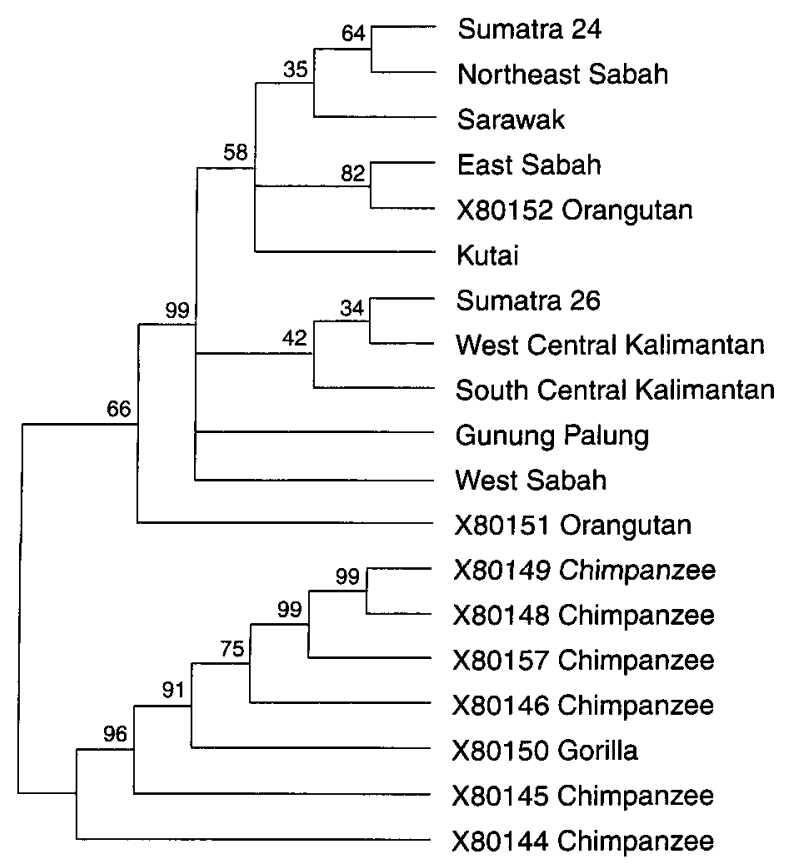

Fig. 3. Single most parsimonious solution of a maximum parsimony analysis of the vWF locus for selected hominoids (length $=85 \mathrm{steps}$ ). Numbers above nodes refer to bootstrap frequencies (1000 replicates). 

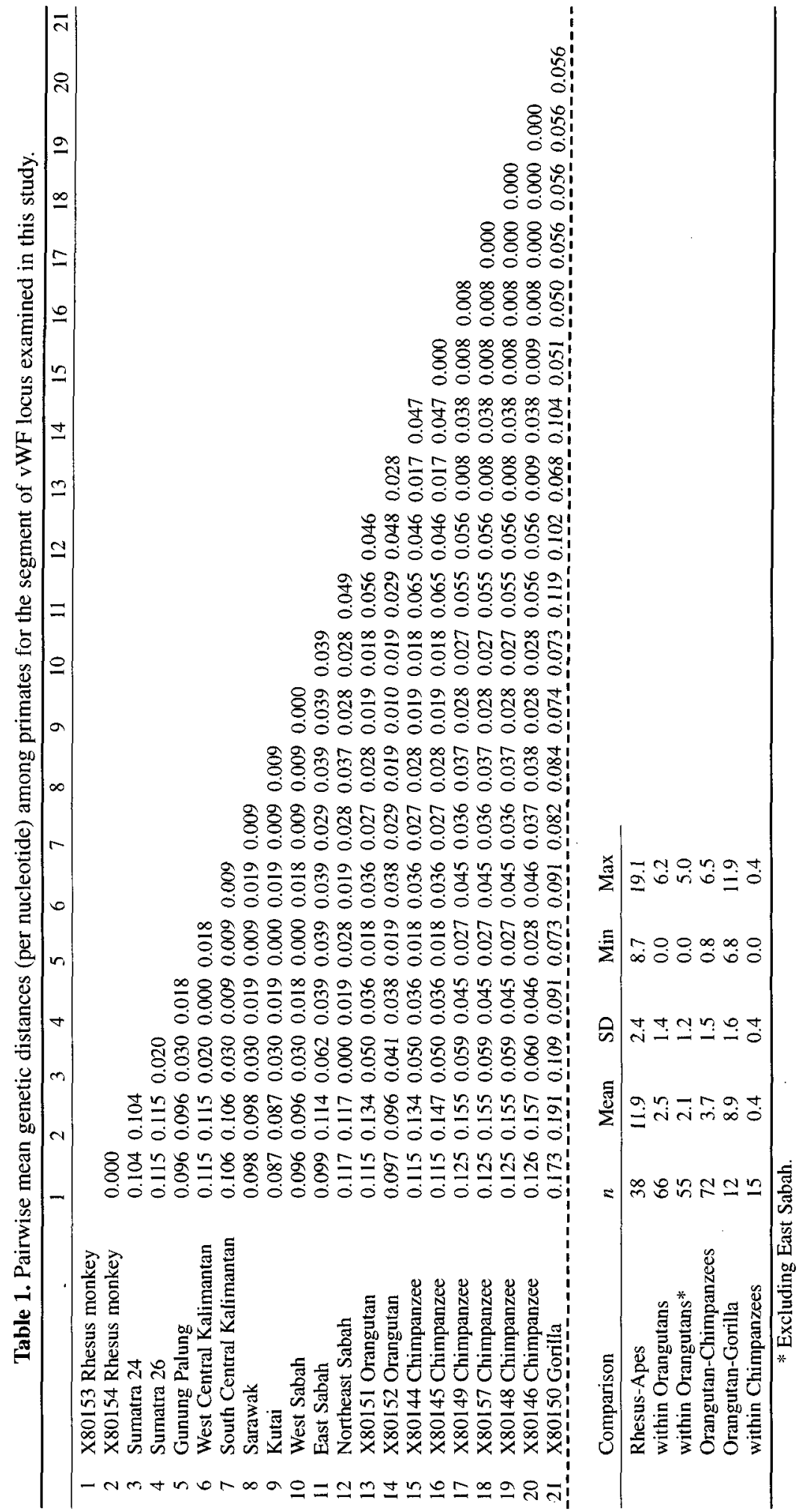
inconspicuous compared to the accumulation of point mutations and indels. This distinction is probably associated with the monomorphic nature of the Pongo vWF locus.

Although the vWF locus is among the most polymorphic of SSR loci in other primate taxa, it is monomorphic in the Pongo. This may be a consequence of the smaller number of repeats in orangutans, in accordance with the often reported positive correlation between repeat count and level of polymorphism (GOLDSTEIN \& CLARK, 1995). In this study, all four species are represented by compound and interrupted SSR alleles with significantly diminished tandemly repeated motifs due to the accumulation of mutations (JARNE \& LAGODA, 1996). The incorporation of mutations and the occurrence of sizable deletions (a repeat motif or more) in combination with stepwise reduction to shorter lengths, might lead to degradation and loss of variance in length that eventually results in the locus becoming monomorphic (COLSON \& GOLDSTEIN, 1999). Interestingly, this degradation does not seem to have affected the variability of this locus in rhesus monkeys: fragment length heterozygosity estimates are between 0.69 (MoRIN et al., 1997) and 0.85 (MEYER et al., 1995). The low GC content observed within this locus is also characteristic of a selectively neutral region, a likely area for large deletions (GLENN et al., 1996; Petrov \& HartL, 1998) that can also contribute to the loss of length polymorphism. Conversely, at the population level, the monomorphic vWF locus in orangutans may reflect a low rate of mutation by the concurrent increase and decrease of array lengths. The $100 \%$ SSR fragment length homozygosity at the orangutan vWF locus may also be suggestive of a selection for sequence length conservation (BOWCOCK et al., 1994) or the combinatorial effect of this type of selection with recent population bottleneck events at that locus. The idea of bottlenecks, however, contrasts with $\mathrm{ZHI}$ et al.'s (1996) conclusion that there have been no significant bottleneck or founder effects in orangutans since the Pleistocene.

MEYER et al. (1995) outlined two serious constraints on polymorphic SSR loci that monomorphic loci, including $\mathrm{VWF}$ in orangutans, may have avoided. Because polymorphic loci are multiallelic in the focal taxon, humans in this case, there may be a bias toward an increased array length in humans and taxa closely related to humans (GOLDSTEIN \& POLLOCK, 1997). Moreover, the process of expansion and contraction of such loci is constantly occurring; and, arrays in different taxa may be at a different point of this process making it difficult to compare homologous loci in different taxa. The fact that the vWF fragments studied here consist solely of homoduplexes also facilitates obtaining and interpreting sequence data without extensive technical hardships. Furthermore, the $v W F$ homoduplexes are reminiscent of generations of population isolation and, consequently bear appreciable genetic information.

The overlap of pairwise mean genetic distances (per nucleotide) between and among taxa may be suggestive of homoplasy not only in terms of sequence length (GARZA \& FrEIMER, 1996) but also in arrangement of repeat arrays and indels. SSR evolution under range constraints (POLLOCK et al., 1998) or variations in the flanking regions (Grimaldi \& CROUAU-RoY, 1997) may also increase the propensity for homoplasy. GARZA and FrEIMER (1996), while examining sequence data from compound SSR loci noted high levels of homoplasy for size both within and between chimpanzees and humans. Similarly, homoplasy among the geographically isolated orangutans cannot be ruled out. However, as evidenced from the observed genetic distances, the genetic differentiation among Bornean Orangutans is at times not only considerably larger than that between the Bornean and Sumatran Orangutans but is also larger than that between Pongo-chimpanzee and gorilla-Pongo pairs.

It may seem surprising that there is such considerable sequence divergence among the Bornean Orangutans mainly among Sabah individuals, among the Gunung Palung and Kalimantan individuals and between the Sumatran individuals. Our observation contrasts with $Z_{H 1}$ et al. (1996) who reported a lack of substantial molecular genetic differentiation among the 
Bornean subpopulations. However, our observations agree with that of another study involving several markers on the orangutan Y chromosome (ALTHEIDE, T. K., pers. comm.) which showed not only a higher level of differentiation among orangutans in Borneo than in Sumatra but also higher diversity within Pongo than within chimpanzees, bonobos or gorillas. Genetic distance estimates among populations vary with different gene families and molecular systematic techniques. Our findings suggest that the orangutans in this study represent populations that may have been isolated due to a lack of gene flow and to subsequent allopatric speciation. These subpopulations are thus genetically unique. It is apparent that the diversity of autochthonous vWF sequences was propagated or preserved due to the presence of strong genetic drift and the absence of migration. The Sumatran sequences differ significantly from each other mainly due to the large deletion in Sumatra 24 sequence. Unfortunately, the lack of pedigree information prevents the verification that the deletion is a rare artifact, vis a vis multigenerational inheritance, questioning its implication for the overall substructuring of the Sumatran populations.

Our study qualifies the conclusions of JANCZEWSKI (1989); JANCZEWSKI et al. (1990); RYDER and ChEMNick (1993); Xu and ARNASON (1996); ZhI et al. (1996); and Kanthaswamy et al. (in press) which supported species level distinction between the Sumatran and Bornean populations. The significantly higher levels of divergence among the orangutans than between the African apes suggests that extant orangutan subpopulations are genetically isolated from each other. Collectively, the results imply significant genetic differentiation, probably at the subspecies level, among the Bornean subpopulations. These subpopulations may have been genetically differentiated even before their final physical separation, perhaps by earlier events leading to isolation. Reticulation between some Sumatran and Bornean Orangutan lineages or homoplasy may have caused the failure of both island populations to fall into two distinct clades. Moreover, a tree that is built using only one gene from each taxa does not necessarily have to be in congruence with the species tree $(\mathrm{NEI}, 1988)$. Further studies of longer sequences at this locus, of additional monomorphic loci and of a larger number of individuals are necessary to select among these inferences. In the interim, the genetic imperative for orangutan conservation management in Borneo and Sumatra should continue to employ readily available molecular and conventional genetic tools.

Acknowledgements. We thank Dr. Mike SyVANEN for his advice and comments on the manuscript. We thank the following facilities for providing us with samples ex gratia: Stephen J. O'Brien Laboratory, Laboratory of Genomic Diversity, National Cancer Institute at Frederick Cancer Research and Development, Frederick, MD; Auduborn Zoo, New Orleans, LA; Birmingham Zoo, Alabama Zoological Society, Birmingham, AL; Cheyene Mountain Zoo, Colorado Springs, CO; Brookfield Zoo, Chicago Zoological Society, Brookfield, IL; Marine World USA, Vallejo, CA; Metro Zoo, Miami, Florida; San Francisco Zoological Garden, San Francisco, CA; St. Louis Zoo, Woodland Park Zoological Gardens, Seattle, WA; Yerkes Primate Center, Emory University, Atlanta, GA; Center for Reproduction of Endangered Species (CRES), San Diego Zoo, San Diego, CA. This study was supported by grants RR00169 and RR05090 from the National Institutes of Health. Bininda-Emonds was supported by an NSERC Postdoctoral Fellowship.

\section{REFERENCES}

Amos, B. C.; Schlotterer, C.; TAutz, D. 1993. Social structure of Pilot whales revealed by analytical DNA profiling. Science, 260: 670-672.

Bowcock, A. M.; Ruiz-Lineares, A.; Tomfohrde, J.; Minch, E.; Kidd, J. R.; Cavalli-Sforza, L. L. 1994. High resolution of human evolutionary tree with polymorphic microsatellites. Nature, 368: $455-$ 457 . 
Bruford, M. W.; Cheeseman, D. J.; Coote, T.; Green, H. A. A.; Haines, S. A.; O'Ryan, C.; Williams, T. R. 1996. Microsatellites and their application in conservation genetics. In: Molecular Genetics Approaches to Conservation, Smith, T. B.; Wayne, R. K. (eds.), Oxford Univ. Press, New York, pp. $278-297$.

Colson, I.; Goldstern, D. B. 1999. Evidence for complex mutations at microsatellite loci in Drosophila. Genetics, 152: $617-627$.

DALLAS, J. F. 1992. Estimation of microsatellite mutation rates in recombinant inbred strains of mouse. Mamm. Genome, 3: 452 - 456.

Deka, R.; Shriver, M. D.; Yu, L. M.; Jin, L.; Aston, C. E.; Chakraborty, R.; Ferrel, R. E. 1994. Conservation of human chromosome 13 polymorphic microsatellite $(\mathrm{CA})_{\mathbf{n}}$ repeats in chimpanzees. Genomics, 22: $226-230$.

Ely, J.; Aivaliotis, M. J.; Kalmin, B.; Manis, G. S.; Reeves-Daniel, A.; Vandeberg, J.; Stone, W. H. 1999. Comparison of biochemical polymorphisms and short tandem repeat (STR) DNA markers for paternity testing in rhesus monkeys (Macaca mulatta). Biochem. Genet., 12 (11/12): 323 - 334.

Felsenstein, J. 1985. Confidence limits on phylogenies: an approach using the bootstrap. Evolution, 39: $783-791$.

Garza, J. C.; Freimer, N. B. 1996. Homoplasy for size at microsatellite loci in human and chimpanzees. Genome Res., 6: 211 - 217.

Glenn, T. C.; Stephan, W.; Dessauer, H. C.; Braun, M. J. 1996. Allelic diversity in alligator microsatellite loci is negatively correlated with GC content of flanking sequences and evolutionary conservation of PCR ampllifiability. Mol. Biol. Evol., 13: 1151 - 1154.

Goldstein, D. B.; Clark, A. G. 1995. Microsatellite variations in North American populations of Drosophila melanogaster. Nucleic Acid Res., 23: 3882 - 3886.

GoldsteIN, D. B.; Pollock, D. D. 1997. Launching microsatellites: a review of mutation processes and methods of phylogenetic inference. Heredity, 88: $335-342$.

Grimaldi, M. C.; Crouau-Roy, B. 1997. Microsatellite allelic homoplasy duc to variable flanking sequences. J. Mol. Evol., 44: 336 - 340.

JANCZEWSKI, D. N. 1989. Estimate of genetic distance of orangutan (Pongo pygmaeus) subspecies based on isozyme and two-dimensional gel electrophoresis. Ph.D. thesis, George Mason Univ., Virginia.

JanCZewSKi, D. N.; Goldman, D.; O'Brien, S. J. 1990. Molecular genetic divergence of orangutan (Pongo pygmaeus) subspecies based on isozyme and two-dimensional electrophoresis. Heredity, 81: $375-387$.

JARNE, P.; LAGODA, P. J. L. 1996. Microsatellites, from molecules to populations and back. Tree, 10: 424 429.

Kanthaswamy, S.; Smith, D. G. 1998. Use of microsatellite polymorphisms for paternity exclusion in rhesus macaques (Macaca mulatta). Primates, 39: 135- 145.

Kanthaswamy, S.; Smith, D. G.; Ely, J. J. in press. Use of microsatellite polymorphisms for the conservation management of wild and feral orangutans. In: Third International Great Apes of the World Conference, Kuching, Malaysia, July 1998.

Kitching, I. J.; Forey, P. L.; Humphries, C. J.; Williams, D. M. 1998. Cladistics: The Theory and Practice of Parsimony Analysis (2nd ed.). The Systematics Association Publication No. 11. Oxford Univ. Press, Oxford.

LewIN, B. 1997. Genes VI. Oxford Univ. Press, New York.

Mancuso, D. J.; Tuley, E. A.; Westfield, L. A.; Worrall, N. K.; Shelton-Inloes, B. B.; Sorrace, J. M.; AleCY, Y. G.; SAdLER, N. C. 1989. Structure of the human von Willebrand factor. J. Biol. Chem., 264: $19514-19527$.

Meyer, E.; Wiegand, P. R.; Rand, S. P.; Kuhlmann, D.; Brack, M.; Brinkmann, B. 1995. Microsatellite polymorphism reveal phylogenetics relationship in primates. J. Mol. Evol., 41: $10-14$.

Moller, A.; Meyer, E.; Brinkmann, B. 1994. Different types of structural variation in STRs: HumFES/FPS, HumVWA and HumD21S11. Int. J. Legal Med, 106: 319 -323.

Morin, P. A.; Kanthaswamy, S.; Smith, D. G. 1997. Simple sequence repeat (SSR) polymorphisms for colony management and population genetics in rhesus macaques (Macaca mulatta). Amer: $J$. Primatol., 42: $199-213$.

Morin, P. A.; SMITH, D. G. 1995. Nonradioactive detection of hypervariable simple sequence repeats in short polyacrylamide gels. Biotechniques, 19: $223-227$.

MorIN, P. M.; WOODRUFF, D. S. 1992. Noninvasive genotyping for vertebrate conservation. In: Molecular Genetics Approaches to Conservation, SMITH, T. B.; WAYNE, R. K. (eds.), Oxford Univ. Press, New York, pp. $298-313$. 
NeI, M. 1988. Molecular Evolutionary Genetics. Columbia Univ. Press, New York.

Nichols, R. A.; BALDING, D. J. 1991. Effects of population structure on DNA fingerprinting analysis in forensic science. Heredity, 66: $297-302$.

Petrov, D. A.; HARTL, D. L. 1998. High rate of DNA loss in the Drosophila melanogaster and Drosophila virilis species groups. Mol. Biol. Evol., 15: 293 - 302.

Pollock, D. D.; Bergman, A.; Feldman, M. W.; Goldstein, D. B. 1998. Microsatellite behavior with range constraints: parameter estimation and improved distances for use in phylogenetic reconstruction. Theoretical Population Biol., 53: $256-271$.

Ryder, O. A.; CheMnick, L. G. 1993. Chromosomal and mitochondrial DNA variation in orangutans. Heredity, 84: 405 - 409.

Saiki, R. K.; Gelfand, D. H.; Stoffel, S.; Scharf, S. J.; Higuchi, R. H.; Hom, G. T.; Mullis, K. B.; ERLICH, E. R. 1988. Primer-directed amplification of DNA with a thermostable DNA polymerase. Science, 239: 487 - 491.

Smith, D. G.; Kanthaswamy, S.; Disbrow, M.; Wagner, J. L. 1999. Reconstruction of parentage in a band of captive Hamadryas baboons. Int. J. Primatol., 20: 415 - 429.

SwOFFord, D. L. 1999. PAUP* [Phylogenetic Analysis Using Parsimony ( ${ }^{*}$ and Other Methods)]. Version 4. Sinauer Associates, Sudnerland, Massachusetts.

TAUTz, D. 1989. Hypervariability of simple sequence as a general source of polymorphic markers. Nuc. Acids Res., 17: 6463 - 6471 .

Templeton, A. R. 1998. Human races: a genetic and evolutionary perspective. Amer. Anthropologist, 100: $632-650$.

WEBER, J. L.; MAY, P. E. 1989. Abundant class of human DNA polymorphisms which can be typed using the polymerase chain reaction. Amer. J. Human Genet., 44: $388-396$.

Weber, J. L.; WONG, C. 1993. Mutation of human short tandem repeats. Human Mol. Genet., 2: 1123 1128 .

Xu, X.; Arnason, U. 1996. The mitochondrial DNA molecule of Sumatran orangutan and a molecular proposal for two (Bornean and Sumatran) species of orangutan. J. Mol. Evol., 3: 431 - 437.

Zhi, L.; Karesh, W. B.; Janczewski, D. N.; Frazier-TaYlor, H.; Dondin Sajuthi; Francis Gombek; Mahedi Andau; Martenson, J. S.; O'Brien, S. J. 1996. Genomic differentiation among natural populations of orangutan (Pongo pygmaeus). Cur. Biol., 6: 1326 - 1336.

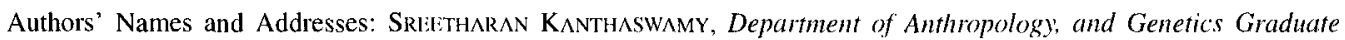
Group, University of California, Davis, One Shields Avenue, Davis, California 95616, U. S. A. e-mail: skkanthaswamy@ucdavis.edu; O. R. P. Binibn-Emonds, Section of Evolution and Ecology, University of California, Davis, One Shields Avenue, Davis, California 956J6, U. S. A.; C. WARdin, Rowe Genetics Program, School of Medicine, University of California, Davis, One Shields Avenue, Davis, California 95616, U. S. A.; J. L. VIRAY, Department of Anthropology, and Genetics Graduate Group, University of California, Davis, One Shields Avenue, Davis, Califormia 956I6, U. S. A.; D. G. SMITH, Department of Anthropology, and California Regional Primate Research Center; University of California, Davis, One Shields Avenue, Davis, California 95616, U. S. A. 